University of Warwick institutional repository

This paper is made available online in accordance with

publisher policies. Please scroll down to view the document

itself. Please refer to the repository record for this item and our

policy information available from the repository home page for further information.

To see the final version of this paper please visit the publisher's website. Access to the published version may require a subscription.

Author(s): Ai Hui Tan, Keith R. Godfrey, and H. Anthony Barker

Article Title: Design of Ternary Signals for MIMO Identification in the Presence of Noise and Nonlinear Distortion

Year of publication: 2009

Link to published version :

http://dx.doi.org/ 10.1109/TCST.2009.2012424

Publisher statement: none 


\title{
Design of Ternary Signals for MIMO Identification in the Presence of Noise and Nonlinear Distortion
}

\author{
Ai Hui Tan, Keith R. Godfrey, and H. Anthony Barker
}

\begin{abstract}
A new approach to designing sets of ternary periodic signals with different periods for multi-input multi-output system identification is described. The signals are pseudo-random signals with uniform nonzero harmonics, generated from Galois field GF $(q)$, where $q$ is a prime or a power of a prime. The signals are designed to be uncorrelated, so that effects of different inputs can be easily decoupled. However, correlated harmonics can be included if necessary, for applications in the identification of ill-conditioned processes. A design table is given for $q \leq 31$. An example is presented for the design of five uncorrelated signals with a common period $N=168$. Three of these signals are applied to identify the transfer function matrix as well as the singular values of a simulated distillation column. Results obtained are compared with those achieved using two alternative methods.
\end{abstract}

Index Terms-Frequency domain design, multi-input multioutput (MIMO) systems, pseudo-random signals, system identification, uncorrelated signals.

\section{INTRODUCTION}

$\mathbf{F}$ OR the identification of multi-input multi-output (MIMO) systems, it is desirable that the effects of different inputs can be separated to ensure identifiability. Two approaches are commonly applied. The first approach uses shifted versions of the same signal to perturb different inputs, and subsequently separate their effects using cross correlation methods. The second approach makes use of uncorrelated signals so that the effects of different inputs can be decoupled. The latter approach reduces the overall time taken to perform the identification experiment compared with the former approach, and also ensures that the system is not operating under different conditions, which is possible if inputs are perturbed sequentially.

Several methods to design periodic perturbation signals which are uncorrelated have been proposed in the existing literature, and are reviewed in Section II. Among the popular methods are the "zippered spectrum" approach and that of modulating a signal with rows of a Hadamard matrix to generate further signals in the set. The former approach allows signal sets to be designed with very few constraints on the signal period, but suffers from the fact that a large number of input levels are required. On the other hand, the latter approach usually requires a very long common period for the signal set,

Manuscript received April 04, 2008; revised November 13, 2008. Manuscript received in final form January 02, 2009. First published April 21, 2009; current version published June 24, 2009. Recommended by Associate Editor D. Rivera. This work was supported in part by the Ministry of Science, Technology, and Innovation, Malaysia under Science Fund Grant 03-02-01-SF004.

A. H. Tan is with the Faculty of Engineering, Multimedia University, 63100 Cyberjaya, Malaysia (e-mail: htai@mmu.edu.my).

K. R. Godfrey is with the School of Engineering, University of Warwick, Coventry, CV4 7AL, U.K. (e-mail: k.godfrey@warwick.ac.uk).

H. A. Barker is with the School of Engineering, Swansea University, Swansea, SA2 8PP, U.K. (e-mail: h.a.barker@swan.ac.uk).

Digital Object Identifier 10.1109/TCST.2009.2012424 but can be applied to signals with a small number of levels, including binary signals.

In this paper, a new approach is proposed which combines the advantages of these two popular methods. This new approach allows a "zippered spectrum" to be realized using sets of ternary signals. Such a design is robust against the effects of both noise and nonlinear distortion, as will be shown in Section VII. Extension to the case of "modified zippered spectrum" is discussed in Section VI.

\section{ReVIEW OF Previous ApProaches}

\section{A. Design of Uncorrelated Signals With the Same Period}

For many years, it was thought that it is not possible to derive uncorrelated periodic signals with the same period, but MacWilliams [1] disproved this by designing a class of pairs of uncorrelated binary signals of period $N=2 p^{2}$, where $p$ is a prime. Briggs and Godfrey [2] showed that, for the pair of MacWilliams signals with $N=18$, the autocorrelation functions of both signals were not remotely of impulsive form. The magnitudes of the harmonics of these signals are far from uniform, and one of the signals has nonzero mean, so the signals are unsuitable for most two-input system identification applications.

In [3], the design of nearly-uncorrelated ternary and quaternary signals of the same period was considered. Three pairs of signals with periods $N=6,7$, or 8 were given, but in the two-input two-output system identification example presented there were large differences between the magnitudes of the harmonics used. As the signal periods were very short, there were too few usable harmonics for most system identification applications.

An approach to designing uncorrelated multisine signals with a "zippered spectrum" was suggested in [4]. For applications in ill-conditioned processes, a "modified zippered spectrum" can be used instead, which consists of both uncorrelated and correlated harmonic components [5], [6]. The frequency domain design of multisine signals is very flexible, as they do not have constraints on the number of signal levels. They are considered plant-friendly as actuator move sizes are generally smaller than those used with pseudo-random signals. However, plant-friendliness is application specific, and some systems cannot accept more than a limited number of signal levels. Hence, when such a limitation exists, a pseudo-random signal is considered more plant-friendly due to its ease of implementation. An example is the identification of the frequency response between the applied force and strip position on a scale model of a hot-dip galvanizing process for steel strip [7]. In this application, the strip can be moved by two electromagnets, one on either side, but the associated power electronics only allow one voltage level to be applied to each. The signals applied are limited to a maximum of 
three levels, corresponding to force to the right, force to the left, and no force. A further example is the identification of an electronic nose described in [8]. In this system, there are four compartments which can be filled with different chemicals or the same chemical but with different concentrations. A metal oxide semiconductor sensor is exposed to the content in one of the compartments at any particular time. The input is implemented using four on-off valves. In [8], only two compartments were used but the physical structure of the system allows a maximum of four input levels to be applied.

\section{B. Design of Uncorrelated Signals With Different Periods}

The design of pseudo-random signals with different periods for the identification of MIMO systems was considered by Briggs and Godfrey [9]. By far the most commonly used design has been that of modulating a pseudo-random binary signal (PRBS) by rows of a Hadamard matrix, proposed in [10]. Each of the signals in the set has an autocorrelation function that is approximately impulsive.

More recently, the authors of the present paper have described two types of periodic ternary pseudo-random maximum length (PRML) signals, designed to identify a linear representation of a single-input system that has noise and nonlinear distortion [11]. Common sources of nonlinear distortion in a system include actuator saturation and material property changes across different operating ranges. A considerable amount of work has been reported in the literature on using harmonic suppression to improve the robustness of a signal against nonlinear distortion. Most of this work concentrates on the use of multisine signals, such as [12]-[14]. Following the definition in [11], a Type 1 signal has even harmonics suppressed, so eliminating completely errors in odd-order estimates from even-order distortion and vice versa. Note that it may also have other harmonic multiples suppressed, but not harmonic multiples of three. A Type 2 signal has harmonic multiples of two and three suppressed, with the possibility of other harmonic multiples being suppressed as well, to further reduce errors from nonlinear distortion.

In this paper, the work in [11] is extended to identify MIMO systems. While such a possibility was briefly alluded to in [11], the method of design suggested therein requires the signal periods to be in the ratio $N, 2 N, 4 N, 8 N, \ldots$. The same restriction is observed when signals in a set are designed based on modulation with the rows of a Hadamard matrix. Here, this constraint has been removed by using a different method of design. Other possible combinations of harmonics which were not considered in [11] are also included here. The resulting sets of signals have different periods, but they also share a common period. The possible combinations are dependent on the generating field. This follows the concept of the "zippered spectrum" approach which has hitherto only been possible using computer-optimized signals such as multisines [5], [6], [15].

\section{DESIGN OF PRML SIGNALS FROM GALOIS FIELDS}

A Galois field $\operatorname{GF}(q)$ comprises $q$ elements which may be represented by the integers 0 to $q-1$, where $q$ is either a prime or a power of a prime. A PRML sequence $s_{q, n}(i)$ in $\mathrm{GF}(q)$ has period $N=q^{n}-1$ and is generated from

$$
s_{q, n}(i)=-\sum_{r=1}^{n} c_{r} s_{q, n}(i-r) \quad \text { all } i
$$

[16], where $i$ is a time index, and $c_{r}$ are the coefficients of a primitive polynomial $f_{q, n}(x)$ of order $n$ in $\mathrm{GF}(q)$, given by

$$
f_{q, n}(x)=\sum_{r=0}^{n} c_{r} x^{r} \quad c_{0}=1 ;(-1)^{n} c_{n} \text { primitive. }
$$

In a period $N$, each nonzero field element occurs $q^{n-1}$ times, while the zero element occurs $q^{n-1}-1$ times.

A primitive PRML sequence $s_{q, 1}(i)$ is generated from (1) with $n=1$ and has period $N_{m}=q-1$ [17]. In a period of $s_{q, 1}(i)$, each nonzero field element occurs once, but the zero field element does not occur. A primitive PRML signal $m(i)$, also of period $N_{m}=q-1$, can be obtained from a primitive PRML sequence $s_{q, 1}(i)$ using the signal conversion

$$
m(i)=l\left(s_{q, 1}(i)\right) \quad \text { all } i
$$

[18], where each field element $e$ of $\mathrm{GF}(q)$ is converted into a corresponding signal level $l(e)$. In a period of $m(i)$

$$
m(i)=l\left(g^{i-1}\right) \quad i=1,2, \ldots, q-1
$$

where $g$ is a primitive element of the field, having the important property that its powers $g^{0}, g^{1}, \ldots, g^{q-2}$ generate all the nonzero elements of the field. If the same field element conversions are used to convert a PRML sequence $s_{q, n}(i)$ with $n \neq 1$ into a PRML signal $u(i)$ with the same period $N$, such that

$$
u(i)=l\left(s_{q, n}(i)\right) \quad \text { all } i
$$

then the harmonic properties of $u(i)$ and $m(i)$ are related to one another, provided $q$ is odd and $l(0)=0$. In particular, the magnitudes of the harmonics of $u(i)$, defined through the discrete Fourier transform (DFT) $|U(k)|$ of $u(i)$ as

$$
|U(k)|=\left|\sum_{i=1}^{N} u(i) \exp \left(-\frac{2 \pi \mathrm{j} i k}{N}\right)\right| k=0,1, \ldots, N-1
$$

are related to the magnitudes $|M(k)|$ of the harmonics of $m(i)$ through

$$
\begin{aligned}
|U(k)|= & |M(0)| q^{n-1} \quad k \text { a multiple of } q^{n}-1 \\
|U(k)|= & |M(0)| q^{(n-2) / 2} \\
& k \text { a multiple of } q-1 \text { but not of } q^{n}-1 \\
|U(k)|= & |M(k)| q^{(n-1) / 2} \\
& k \text { not a multiple of } q-1
\end{aligned}
$$

[18]. The set of relationships in (7) therefore provides an efficient and convenient means for the generation of PRML signals as the design can be carried out on their primitive versions which have much shorter periods. 


\section{SIGNAL DESIGN FOR MIMO SYSTEM IDENTIFICATION}

To identify a linear representation of a system that has noise and nonlinear distortion, it is advantageous to minimize the signal amplitude in order to reduce the effects of the latter. At the same time, to achieve a high signal-to-noise ratio, the available power should be increased. These conflicting requirements led to the concept of performance index for perturbation signals (PIPS) [19], defined by

$$
\mathrm{PIPS}=\frac{200\left(u_{\mathrm{rms}}^{2}-u_{\mathrm{mean}}^{2}\right)^{1 / 2}}{u_{\max }-u_{\min }} \%
$$

where $u_{\text {rms }}, u_{\text {mean }}, u_{\text {max }}$, and $u_{\text {min }}$ are, respectively, the root mean square, mean, maximum, and minimum values of the discrete signal $u(i)$. Effectively, PIPS is based on the goal in traditional experiment design which is to obtain the most accurate estimates of the system subject to constraint on the maximum allowable amplitude. PIPS is independent of both the signal mean and amplitude scale. It is $100 \%$ for a signal with the best possible performance, namely a binary signal for which $u_{\max }$ and $u_{\text {min }}$ have equal duration or number of occurrences.

For the ternary signals described in this paper, PIPS is directly related to how the elements in $\operatorname{GF}(q)$ are mapped into signal levels. In particular, for a primitive signal $m(i)$ with $r$ elements converted to $+1, r$ elements converted to -1 and $(q-1-2 r)$ elements converted to 0 , PIPS is given by

$$
\mathrm{PIPS}_{m}=\sqrt{\frac{2 r}{q-1}} \times 100 \% .
$$

For the signal $u(i)$

$$
\begin{aligned}
\operatorname{PIPS}_{u} & =\sqrt{\frac{2 r q^{n-1}}{q^{n}-1}} \times 100 \% \\
& =\sqrt{\frac{q^{n}-q^{n-1}}{q^{n}-1}} \times \mathrm{PIPS}_{m} .
\end{aligned}
$$

In addition to this, it is desirable that the magnitudes of the harmonics in the signal be uniform, in order to uniformly excite the system across the frequency band of interest. Multiples of certain harmonics should also be suppressed for greater robustness against the effects of nonlinear distortion.

One of the methods to design signals with uniform DFT magnitude, and good immunity towards the effects of noise and nonlinear distortion was introduced in [20], where the idea of using primitive signals containing several sub-periods within $N_{m}$ was first proposed. If $q$ is odd, $m(i)$ can be designed such that $m(i)=(-1)^{i-1}, i=1,2, \ldots,(q-1)$ [21]. The resulting signal is Type 1 . If $(q-1)$ is an integer multiple of $6, m(i)$ can be designed such that $m_{q}(i)=\left[m_{7}(i), m_{7}(i), \ldots, m_{7}(i)\right]$, where $m_{7}(i)=[+1,+1,0,-1,-1,0]$ is repeated $(q-1) / 6$ times to give $m_{q}(i)$ [22]. The resulting signal is Type 2 .

In this paper, the idea of allowing multiple periods within a common period $N_{m}$ has been extended, with a search carried out across different permutations of the signal levels in the first half period of $m(i)$. The search is carried out separately for signals in which the even harmonics are zero and the second half of the

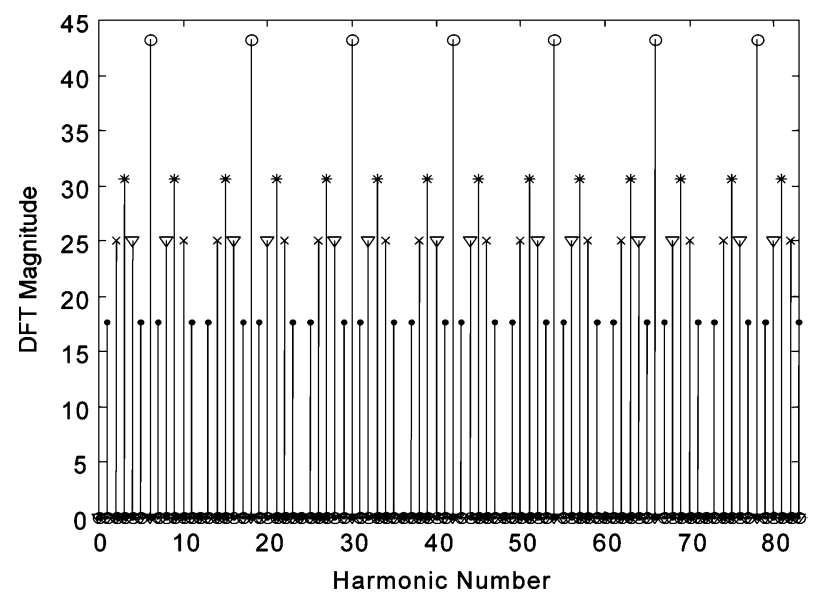

Fig. 1. First half period of the DFT magnitudes of uncorrelated signals from $\mathrm{GF}(13)$, with $n=2$. The primitive signals are $\mathrm{B}$ (dots), $\mathrm{E}$ (crosses), $\mathrm{F}$ (asterisks), $\mathrm{G}$ (triangles), and $\mathrm{H}$ (circles), as given in Table I.

period is the negative of the first half, and for signals in which the odd harmonics are zero and the second half of the period is the same as the first half.

The results are tabulated in Table I, which shows all possible combinations of uncorrelated signals for $q \leq 31$. For certain combinations of nonzero harmonics where there is more than one $m(i)$ that results in a uniform spectrum, the one with the highest $\mathrm{PIPS}_{m}$ is selected. For every signal in a set, the first nonzero harmonic $k$ in Table I must be a factor of $q-1$, and the remaining nonzero harmonics must be integer multiples of the first $k$. No theoretical relationship could be found between $q$ and the possible number of input combinations.

It should be made clear that the common period is not necessarily $q^{n}-1$, but can be a sub-multiple of it. For instance, using $\mathrm{GF}(17)$, if only three uncorrelated signals are needed, then Signals B, C and D with periods 144,72 , and 36, respectively, can be selected from Table I. The common period is therefore 144 and not 288 in this case. This leads to a much larger number of possible common periods designed using the proposed approach.

A step-by-step guideline on how Table I can be applied is given in the following.

1) Based on the number of signals required in a signal set, obtain the possible generating fields from Table I.

2) For each of these fields, calculate the possible common periods. Choose the field for which the common period is closest to the most suitable common period for the application.

3) For the chosen field, if there is more than one possible choice of signals then priority should be given to either Type 1 or Type 2 signals, depending on the application, and remaining signals should be Type $\mathrm{O}$, which have some harmonic suppression, or Type $\mathrm{N}$, which have no harmonic suppression, in that order.

4) If there is still more than one possible combination of signals, then preference should be given to signals with higher PIPS values. However, if it is more important that all signals in a set have similar PIPS values, this can also be chosen accordingly. 
TABLE I

Combinations of Uncorrelated Signals for $q \leq 31$. Only the First Half Period of $m(i)$ is Given. The Notation $\{+\}$ Denotes That the Second Half Period is the Same as the First Half, While $\{-\}$ Denotes That the Second Half Period is the Negative of the First Half

\begin{tabular}{|c|c|c|c|c|c|c|}
\hline$q$ & $m(i)$ & & $\mathrm{PIPS}_{m}(\%)$ & Nonzero harmonics $k$ & $|M(k)|$ & Typ \\
\hline \multirow[t]{3}{*}{5} & $\mathrm{~A}$ & $11\{-\}$ & 100 & 1,3 & 2.83 & \\
\hline & $\mathrm{B}$ & $1-1\{+\}$ & 100 & 2 & 4 & 1 \\
\hline & \multicolumn{6}{|c|}{ Possible combination: $\mathrm{AB}$} \\
\hline 7 & A & $010\{-\}$ & 57.74 & $1,3,5$ & 2 & 1 \\
\hline & $\mathrm{B}$ & $110\{-\}$ & 81.65 & 1,5 & 3.46 & 2 \\
\hline & $\mathrm{C}$ & $1-10\{+\}$ & 81.65 & 2,4 & 3.46 & $\mathrm{~N}$ \\
\hline & $\mathrm{D}$ & $1-11\{-\}$ & 100 & 3 & 6 & 1 \\
\hline & Poss & e combinations: $\mathrm{AC}, \mathrm{BCD}$ & & & & \\
\hline 9 & A & $0111-1\{-\}$ & 86.60 & $1,3,5,7$ & 3.46 & 1 \\
\hline & B & $11-1-1\{+\}$ & 100 & 2,6 & 5.66 & 1 \\
\hline & $\begin{array}{ll}\mathrm{C} \\
\mathrm{n}\end{array}$ & $1-11-1\{+\}$ & 100 & 4 & 8 & 1 \\
\hline & Possi & e combination: $\mathrm{ABC}$ & & & & \\
\hline 11 & A & $000001\{-\}$ & 44.72 & $1,3,5,7,9$ & 2 & 1 \\
\hline & B & $1-1-10-1\{-\}$ & 89.44 & $1,3,7,9$ & 4.47 & 1 \\
\hline & $\mathrm{C}$ & $1-1-110\{+\}$ & 89.44 & $2,4,6,8$ & 4.47 & $\mathrm{~N}$ \\
\hline & $\mathrm{D}$ & $1-11-11\{-\}$ & 100 & 5 & 10 & 1 \\
\hline & Poss & e combinations: $\mathrm{AC}, \mathrm{BCD}$ & & & & \\
\hline 13 & A & $01-11111\{-\}$ & 91.29 & $1,3,5,7,9,11$ & 4.47 & 1 \\
\hline & B & $0011101-1\{-\}$ & 81.65 & $1,5,7,11$ & 4.90 & 2 \\
\hline & $\mathrm{C}$ & $10-10000\{+\}$ & 57.74 & $2,4,8,10$ & 3.46 & $\mathrm{O}$ \\
\hline & $\mathrm{D}$ & $100-100\{+\}$ & 57.74 & $2,6,10$ & 4 & 1 \\
\hline & $\mathrm{E}$ & $110-1-10\{+\}$ & 81.65 & 2,10 & 6.93 & 2 \\
\hline & $\mathrm{F}$ & $11-1-111\{-\}$ & 100 & 3,9 & 8.49 & 1 \\
\hline & G & $01-101-1\{+\}$ & 81.65 & 4,8 & 6.93 & $\mathrm{~N}$ \\
\hline & $\mathrm{H}$ & $1-11-11-1\{+\}$ & 100 & 6 & 12 & 1 \\
\hline & Possi & e combinations: $\mathrm{ACH}, \mathrm{ADG}, \mathrm{AEGH}, \mathrm{BCFH}$ & FG, BEFGH & & & \\
\hline 17 & A & $1-1-1-10-11-1\{-\}$ & 93.54 & $1,3,5,7,9,11,13,15$ & 5.29 & 1 \\
\hline & $\mathrm{B}$ & $1-1-10-1110\{+\}$ & 86.60 & $2,6,10,14$ & 6.93 & 1 \\
\hline & $\mathrm{C}$ & $1-1-1111-1-11\{+\}$ & 100 & 4,12 & 11.31 & 1 \\
\hline & $\mathrm{D}$ & $1-11-11-11-1\{+\}$ & 100 & 8 & 16 & 1 \\
\hline & Poss & e combination: $\mathrm{ABCD}$ & & & & \\
\hline 19 & A & $100000000\{-\}$ & 33.33 & $1,3,5,7,9,11,13,15,17$ & 2 & 1 \\
\hline & B & $100100000\{-\}$ & 47.14 & $1,5,7,11,13,17$ & 3.46 & 2 \\
\hline & $\mathrm{C}$ & $1000-10000000\{+\}$ & 47.14 & $2,4,8,10,14,16$ & 3.46 & $\mathrm{O}$ \\
\hline & $\mathrm{D}$ & $100-1000100\{-\}$ & 57.74 & $3,9,15$ & 6 & 1 \\
\hline & E & $10-1-101110-1\{-\}$ & 81.65 & 3,15 & 10.39 & 2 \\
\hline & F & $1-10101-10101-10\{+\}$ & 81.65 & 6,12 & 10.39 & $\mathrm{~N}$ \\
\hline & G & $1-11-111-11-111\{-\}$ & 100 & 9 & 18 & 1 \\
\hline & Possi & e combinations: ACF, BCDF, BCEFG & & & & \\
\hline 23 & A & $10000000000\{-\}$ & 30.15 & $1,3,5,7,9,11,13,15,17,19,21$ & 2 & 1 \\
\hline & B & $1-1-1-1001111-1111\{-\}$ & 95.35 & $1,3,5,7,9,13,15,17,19,21$ & 6.63 & 1 \\
\hline & $\mathrm{C}$ & $111-1110-111-1-1-1\{+\}$ & 95.35 & $2,4,6,8,10,12,14,16,18,20$ & 6.63 & $\mathrm{~N}$ \\
\hline & $\mathrm{D}$ & $1-11-11-111-11-11\left\{\begin{array}{l}0 \\
0\end{array}\right.$ & 100 & 11 & 22 & 1 \\
\hline & Poss & e combinations: $\mathrm{AC}, \mathrm{BCD}$ & & & & \\
\hline 25 & A & $1-1-1-1-10-11-1111-1\{-\}$ & 95.74 & $1,3,5,7,9,11,13,15,17,19,21,23$ & 6.63 & 1 \\
\hline & B & $1-10010100110\{-\}$ & 70.71 & $1,5,7,11,13,17,19,23$ & 6 & 2 \\
\hline & $\mathrm{C}$ & $1001-11100-1-1-1\{+\}$ & 81.65 & $2,4,8,10,14,16,20,22$ & 6.93 & $\mathrm{O}$ \\
\hline & $\mathrm{D}$ & $11-110-1-1-11-101\{+\}$ & 91.29 & $2,6,10,14,18,22$ & 8.94 & 1 \\
\hline & E & $101-10-1-10-1101\{+\}$ & 81.65 & $2,10,14,22$ & 9.80 & 2 \\
\hline & $\mathrm{F}$ & $1-1-10-111101-1-10\{-\}$ & 86.60 & $3,9,15,21$ & 10.39 & 1 \\
\hline & $\mathrm{G}$ & $10000-1010000-10\{+\}$ & 57.74 & $4,8,16,20$ & 6.93 & $\mathrm{O}$ \\
\hline & $\mathrm{H}$ & $100-100100-100\{+\}$ & 57.74 & $4,12,20$ & 8 & 1 \\
\hline & I & $110-1-10110-1-10\{+\}$ & 81.65 & 4,20 & 13.86 & 2 \\
\hline & $\mathrm{J}$ & $11-1-1111-1-1111-1-1\{+\}$ & 100 & 6,18 & 16.97 & 1 \\
\hline & $\mathrm{K}$ & $10-110-110-110-1\{+\}$ & 81.65 & 8,16 & 13.86 & $\mathrm{~N}$ \\
\hline & $\mathrm{L}$ & $1-11-11-11-11-11-1\{+\}$ & 100 & 12 & 24 & 1 \\
\hline & $\begin{array}{l}\text { Possi } \\
\text { BEFI }\end{array}$ & $\begin{array}{l}\text { e combinations: ACJL, ADGL, ADHK, AD } \\
<L\end{array}$ & AEGJL, AEI & JK, AEIJKL, BCFJL, BDFGL, BDFHK, BDFIKL, & $\mathrm{BJL}, \mathrm{BE}$ & \\
\hline 27 & A & 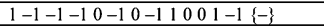 & 83.21 & $1,3,5,7,9,11,13,15,17,19,21,23,25$ & 6 & 1 \\
\hline & $\mathrm{B}$ & $1-1-1-10-1-1-1 \quad 11-11-1\{-\}$ & 96.08 & $1,3,5,7,9,11,15,17,19,21,23,25$ & 7.21 & 1 \\
\hline & $\mathrm{C}$ & 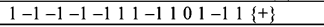 & 96.08 & $2,4,6,8,10,12,14,16,18,20,22,24$ & 7.21 & $\mathrm{~N}$ \\
\hline & $\mathrm{D}$ & $1-11-11-111-11-11-11\left\{\begin{array}{l}1 \\
-1\end{array}\right.$ & 100 & 13 & 26 & 1 \\
\hline & Poss & e combinations: $\mathrm{AC}, \mathrm{BCD}$ & & & & \\
\hline 29 & A & $1-1-1-1-1-1001-111-1111-1\{-\}$ & 96.36 & $1,3,5,7,9,11,13,15,17,19,21,23,25,27$ & 7.21 & 1 \\
\hline & $\mathrm{B}$ & $1-1-1-10-11-1-11-10-1-1\{-\}$ & 92.58 & $1,3,5,9,11,13,15,17,19,23,25,27$ & 7.48 & 1 \\
\hline & $\mathrm{C}$ & $10-10-10000101010-10\left\{\begin{array}{l}0 \\
\end{array}\right.$ & 65.47 & $2,4,6,8,10,12,16,18,20,22,24,26$ & 5.29 & $\mathrm{O}$ \\
\hline & $\mathrm{D}$ & $1-1-100-10-111100010\{+\}$ & 75.59 & $2,6,10,14,18,22,26$ & 8 & 1 \\
\hline & E & $1-1-1-1-1110-111111-10\{+\}$ & 92.58 & $2,6,10,18,22,26$ & 10.58 & 1 \\
\hline & $\mathrm{F}$ & 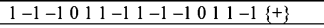 & 92.58 & $4,8,12,16,20,24$ & 10.58 & $\mathrm{~N}$ \\
\hline & $\mathrm{G}$ & $1-1-111-1-1111-1-1111-1\{-\}$ & 100 & 7,21 & 19.80 & 1 \\
\hline & $\mathrm{H}$ & $1-11-11-11-11-11-11-1\{+\}$ & 100 & 14 & 28 & 1 \\
\hline & Poss & e combinations: $\mathrm{ACH}, \mathrm{ADF}, \mathrm{AEFH}, \mathrm{BCGH}$ & FG, BEFGH & & & \\
\hline 31 & A & $100000000000000\{-\}$ & 25.82 & $1,3,5,7,9,11,13,15,17,19,21,23,25,27,29$ & 2 & 1 \\
\hline & $\mathrm{B}$ & $1000-1000-10000000-100 \begin{array}{lll}-1 & 0\end{array}$ & 51.64 & $1,3,7,9,11,13,17,19,21,23,27,29$ & 4.47 & 1 \\
\hline & $\mathrm{C}$ & $1-1-1-10000-1000-110010\{-\}$ & 73.03 & $1,5,7,11,13,17,19,23,25,29$ & 6.93 & 2 \\
\hline & $\mathrm{D}$ & $1-10-100-1-100-10-110 \quad\{-\}$ & 73.03 & $1,7,11,13,17,19,23,29$ & 7.75 & 2 \\
\hline & E & $100-1000-10001000000\{+\}$ & 51.64 & $2,4,6,8,12,14,16,18,22,24,26,28$ & 4.47 & $\mathrm{O}$ \\
\hline & $\mathrm{F}$ & $1-1-10101011000-10000-1\{+\}$ & 73.03 & $2,4,8,10,14,16,20,22,26,28$ & 6.93 & $\mathrm{O}$ \\
\hline & G & $1-10010110-1-10-100\{+\}$ & 73.03 & $2,4,8,14,16,22,26,28$ & 7.75 & $\mathrm{O}$ \\
\hline & $\mathrm{H}$ & $10000-1000010000\{-\}$ & 44.72 & $3,9,15,21,27$ & 6 & 1 \\
\hline & I & $1-1-10-1-11110011-1-10-1\{-\}$ & 89.44 & $3,9,21,27$ & 13.42 & 1 \\
\hline & $\mathrm{J}$ & $100-100100-1000100\{-\}$ & 57.74 & $5,15,25$ & 10 & 1 \\
\hline & $\mathrm{K}$ & $10-1-101110-1-100110-1\{-\}$ & 81.65 & 5,25 & 17.32 & 2 \\
\hline & $\mathrm{L}$ & $1-1-1101-1-1101-1-110\{+\}$ & 89.44 & $6,12,18,24$ & 13.42 & $\mathrm{~N}$ \\
\hline & $\mathrm{M}$ & $1-101-101-101-101-10\{+\}$ & 81.65 & 10,20 & 17.32 & $\mathrm{~N}$ \\
\hline & $\mathrm{N}$ & $1-11-11-11-11-11-11-11\{-\}$ & 100 & 15 & 30 & 1 \\
\hline & & combinations: AEM, AFL, AGLM, BEJN & $\mathrm{MN}, \mathrm{BF}$ & FKLN, BGJLM, BGKLMN, CEH & & LM, \\
\hline
\end{tabular}

\section{DESIGN EXAMPLE}

For the purpose of illustration, a set of uncorrelated signals is generated from $\mathrm{GF}(13)$. Since $q-1=13-1=12$ is divisible by $1,2,3,4$, and 6 , the eight signals in Table I start with one of these as the first nonzero harmonic. There are six different ways these signals can be combined to produce sets of uncorrelated 
signals, but the maximum possible number of inputs in this case is five, where the signals $\mathrm{B}, \mathrm{E}, \mathrm{F}, \mathrm{G}$, and $\mathrm{H}$ are used.

For $\mathrm{GF}(13), g=2$ is a primitive element. Using $g=2$ in (4), the field element conversions for Signal B are $m(1)=l\left(2^{0}\right)=$ $l(1)=0, m(2)=l\left(2^{1}\right)=l(2)=1, m(3)=l\left(2^{2}\right)=l(4)=1$, $m(4)=l\left(2^{3}\right)=l(8)=0, m(5)=l\left(2^{4}\right)=l(3)=1$, $m(6)=l\left(2^{5}\right)=l(6)=-1, m(7)=l\left(2^{6}\right)=l(12)=0$, $m(8)=l\left(2^{7}\right)=l(11)=-1, m(9)=l\left(2^{8}\right)=l(9)=-1$, $m(10)=l\left(2^{9}\right)=l(5)=0, m(11)=l\left(2^{10}\right)=l(10)=-1$, $m(12)=l\left(2^{11}\right)=l(7)=1$. The conversions for the other signals can be obtained in a similar manner.

The magnitudes of the harmonics of this set of five uncorrelated PRML signals generated using $n=2$ are plotted in Fig. 1 using a common period of $N=13^{2}-1=168$. The actual periods are 168 for $\mathrm{B}, 84$ for E, 56 for F, 42 for $\mathrm{G}$, and 28 for H. From (7b), harmonic multiples of 12 are suppressed in all five signals because the corresponding primitive signals have zero mean. From among the five signals, $\mathrm{F}$ and $\mathrm{H}$ are Type 1 signals, with even harmonics suppressed, B and E are Type 2 signals, with harmonic multiples of 2 and 3 suppressed, while $\mathrm{G}$ is neither Type 1 nor Type 2 and would therefore be less suitable for use in a system that has nonlinear distortion. While the harmonics are not uniform across different channels, this is not a problem as different channels are expected to have different gains. If the approximate gains of the channels are known $a$ priori, a signal with higher DFT magnitudes can be selectively applied on a channel with a lower gain.

\section{EXTENSION TO THE IDENTIFICATION OF ILL-CONDITIONED SYSTEMS}

Some MIMO systems are ill-conditioned. This means that they have strong directionality properties and there are large differences between the singular values of the steady-state gain matrix. In the literature, popular approaches for the identification of such systems rely on the use of large amplitude correlated components and small amplitude uncorrelated components. Examples of these approaches can be found in [5], [6], [15], [23].

The ternary signals proposed in the paper can be shaped beyond the standard "zippering". However, it is important to know a priori the directional properties of the system, so that the spectrum can be shaped accordingly. It should be pointed out that pseudo-random ternary signals can in most instances only approximate a required optimum spectrum. This is due to the constraint imposed by the number of signal levels permitted. If the system under study can only accept a limited number of signal levels, then there is a small price to pay in the design of the harmonic spectrum.

An example of a two-input design which consists of both correlated and uncorrelated harmonics is the use of Signals $\mathrm{C}$ and D from $\mathrm{GF}(25)$ in Table I. Here, the correlated harmonics are $2,10,14$, and 22, whereas the uncorrelated ones are 4, 8, 16, and 20 for Signal C, and 6 and 18 for Signal D. The harmonic spectrum of this design is shown in Fig. 2. The total power in each correlated harmonic is much higher than that in each uncorrelated harmonic, due to the power being contributed by both signals in the set.

If it is further required that each individual signal has different amplitudes for correlated and uncorrelated harmonics, a similar

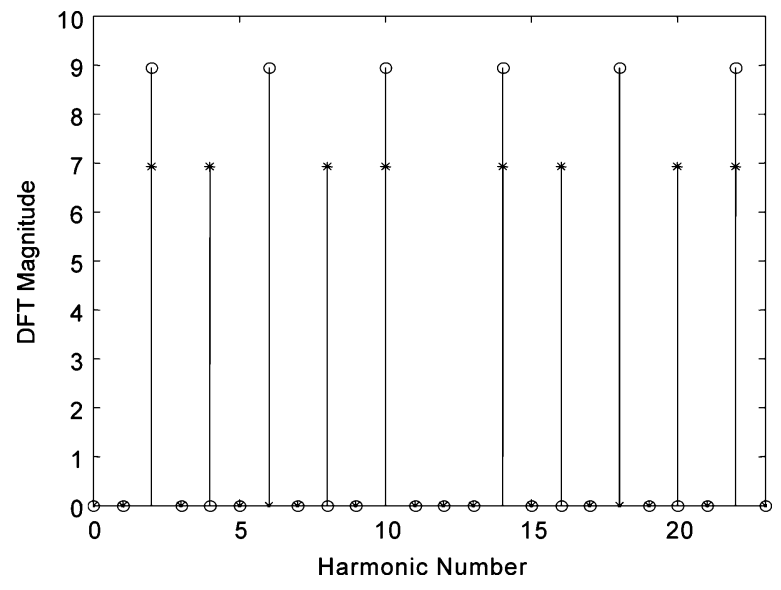

Fig. 2. DFT magnitudes of primitive signals from GF(25). The primitive signals are C (asterisks) and D (circles), as given in Table I.

search procedure as described in Section IV has to be carried out to search for signals with the required spectrum. However, unless the directional properties are known a priori, applying high amplitude correlated harmonics in the wrong direction may cause the system output to exceed permissible bounds [24].

\section{APPliCATION ON A SimUlated Distillation COLUMN}

\section{A. Description of Process and Conditions for Simulation}

A three-input three-output binary ethanol-water system of a pilot-plant distillation column, with a side stream as well as overhead and bottom products, described by Ogunnaike $e t$ al. [25], is simulated. The inputs are reflux flow rate in gpm $\left(x_{1}\right)$, side stream product flow rate in gpm $\left(x_{2}\right)$, and reboiler steam pressure in psig $\left(x_{3}\right)$. The outputs are overhead ethanol mole fraction $\left(y_{1}\right)$, side stream ethanol mole fraction $\left(y_{2}\right)$, and temperature of tray number 19 in degrees Celsius $\left(y_{3}\right)$. The inputoutput relationship, with a time scale in minutes, is given by

$$
\begin{aligned}
{\left[\begin{array}{l}
Y_{1} \\
Y_{2} \\
Y_{3}
\end{array}\right]=} & {\left[\begin{array}{ccc}
\frac{0.66 \mathrm{e}^{-2.6 \mathrm{~s}}}{6.7 \mathrm{~s}+1} & \frac{-0.61 \mathrm{e}^{-3.5 \mathrm{~s}}}{8.64 \mathrm{~s}+1} & \frac{-0.0049 \mathrm{e}^{-\mathrm{s}}}{9.06 \mathrm{~s}+1} \\
\frac{1.11 \mathrm{e}^{-6.5 \mathrm{~s}}}{3.25 \mathrm{~s}+1} & \frac{-2.36 \mathrm{e}^{-3 \mathrm{~s}}}{5 \mathrm{~s}+1} & \frac{-0.01 \mathrm{e}^{-1.2 \mathrm{~s}}}{7.09 \mathrm{~s}+1} \\
\frac{-34.68 \mathrm{e}^{-9.2 \mathrm{~s}}}{8.15 \mathrm{~s}+1} & \frac{46.2 \mathrm{e}^{-9.4 \mathrm{~s}}}{10.9 \mathrm{~s}+1} & \frac{0.87(11.61 \mathrm{~s}+1) \mathrm{e}^{-\mathrm{s}}}{(3.89 \mathrm{~s}+1)(18.8 \mathrm{~s}+1)}
\end{array}\right] } \\
& \times\left[\begin{array}{l}
X_{1} \\
X_{2} \\
X_{3}
\end{array}\right] .
\end{aligned}
$$

The transfer function matrix in (11) will be denoted as $G(s)$ throughout this paper. Singular value decomposition of the steady-state gain matrix gives

$$
\begin{aligned}
G(0)= & U \Sigma V^{T} \\
= & {\left[\begin{array}{ccc}
0.0153 & -0.2917 & 0.9564 \\
0.0442 & 0.9558 & 0.2908 \\
-0.9989 & 0.0378 & 0.0275
\end{array}\right] } \\
& \times\left[\begin{array}{ccc}
57.8377 & 0 & 0 \\
0 & 0.5534 & 0 \\
0 & 0 & 0.0163
\end{array}\right] \\
& \times\left[\begin{array}{ccc}
0.6000 & -0.7988 & 0.0448 \\
-0.7999 & -0.6000 & 0.0148 \\
-0.0150 & 0.0447 & 0.9989
\end{array}\right]^{T}
\end{aligned}
$$




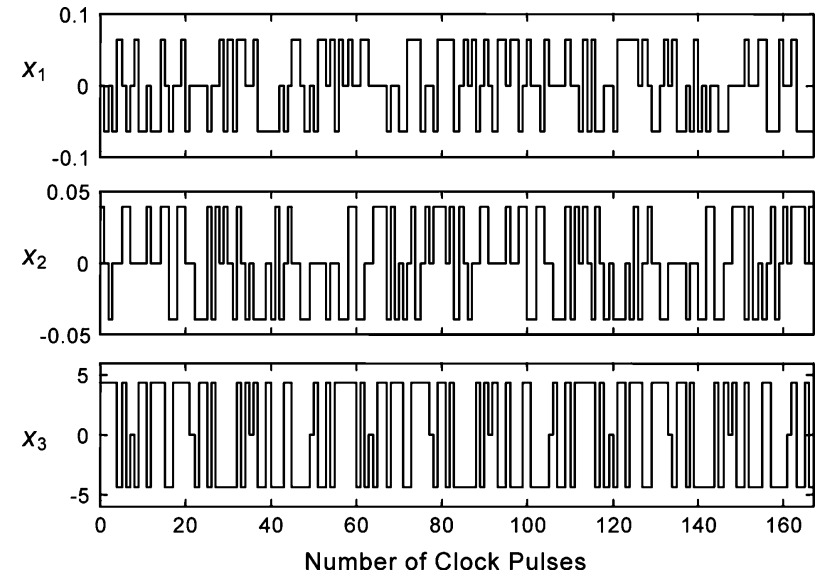

Fig. 3. Inputs $x_{1}, x_{2}$, and $x_{3}$ used in the distillation column application.

where $U$ is the matrix of output singular vectors, $V$ is the matrix of input singular vectors and $\Sigma$ is the diagonal matrix of singular values. The distillation column is a very difficult process to identify, due to the large differences in gain, time constant and time delay. It is also ill-conditioned, as the singular values of $G(0)$ are very different from one another, their values being $\sigma_{1}=57.8377, \sigma_{2}=0.5534$, and $\sigma_{3}=0.0163$. In particular, the high gain input direction is $\left[\begin{array}{lll}0.6000 & -0.7999 & -0.0150\end{array}\right]^{T}$ whereas the low gain input direction is $\left[\begin{array}{lll}0.0448 & 0.0148 & 0.9989\end{array}\right]^{T}$. It is worth noting that while the system is ill-conditioned, it is not highly interactive since the relative gain array $\Lambda$ at steady-state is

$$
\begin{aligned}
\Lambda(0) & =G(0) \circ\left[G^{-1}(0)\right]^{T} \\
& =\left[\begin{array}{ccc}
2.0084 & -0.7220 & -0.2864 \\
-0.6460 & 1.8246 & -0.1786 \\
-0.3624 & -0.1026 & 1.4650
\end{array}\right]
\end{aligned}
$$

where $\circ$ denotes the Schur product.

The only a priori knowledge assumed in the experiment design is that of the smallest and largest time constants in the system, which constrain the choices of the sampling interval $T$ and the measurement period $N T$, respectively, together with the maximum allowable deviations from the usual steady-state values of the system, which constrain the choices of the input signal amplitudes. Based on this, signals $\mathrm{B}, \mathrm{E}$, and $\mathrm{F}$ from $\mathrm{GF}(13)$, with $N=\left(13^{2}-1\right)=168$, were chosen and scaled to amplitudes of $0.065 \mathrm{gpm}, 0.039 \mathrm{gpm}$, and $4.4 \mathrm{psig}$ to form the inputs $x_{1}, x_{2}$, and $x_{3}$, respectively, with a sampling interval $T=0.5 \mathrm{~min}$ and a measurement period $N T=168 \times 0.5=$ 84 min. The inputs are illustrated in Fig. 3. Both Signals B and E have $\mathrm{PIPS}_{m}=81.65 \%$ and $\mathrm{PIPS}_{u}=78.68 \%$, whereas Signal $\mathrm{F}$ has $\mathrm{PIPS}_{m}=100 \%$ and $\mathrm{PIPS}_{u}=96.36 \%$. Since all three signals have high PIPS values, they provide a good balance between the number of nonzero harmonics and the DFT magnitudes at these nonzero harmonics. No knowledge of the directionality properties of the system is assumed and no rotation is applied to any of the input signals.
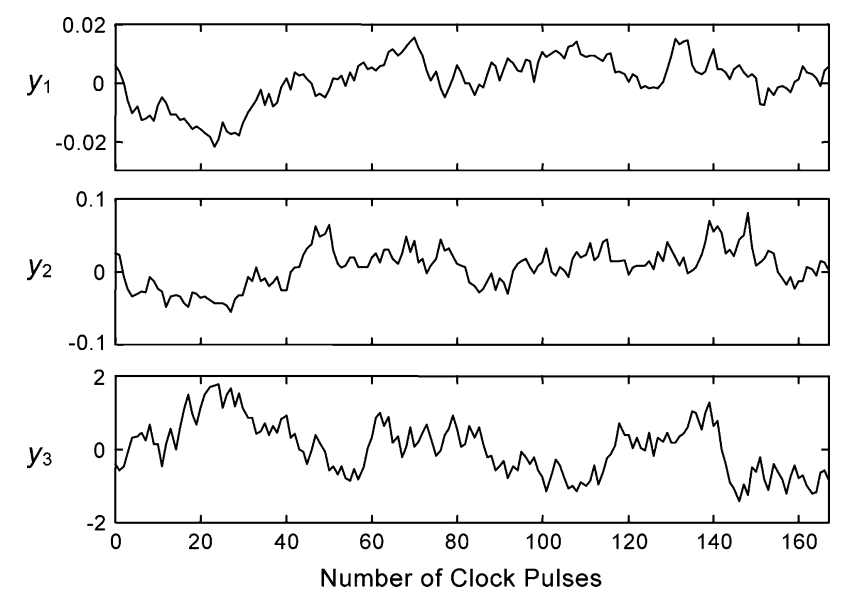

Fig. 4. Outputs $y_{1}, y_{2}$, and $y_{3}$ which are corrupted by noise and nonlinear distortion.

Random noise with zero mean is added to each of the nine input-output channels such that the signal-to-noise ratio for each channel is $15 \mathrm{~dB}$. In order to make the identification problem more challenging, nonlinearity is added to the outputs of each channel. The nonlinear function is

$$
f_{1}(y)=y+10 y^{2}+100 y^{3}
$$

for channels $x_{1} \rightarrow y_{1}, x_{2} \rightarrow y_{1}, x_{3} \rightarrow y_{1}, x_{1} \rightarrow y_{2}, x_{2} \rightarrow y_{2}$, and $x_{3} \rightarrow y_{2}$, but is

$$
f_{2}(y)=y+0.1 y^{2}+0.05 y^{3}
$$

for channels $x_{1} \rightarrow y_{3}, x_{2} \rightarrow y_{3}$, and $x_{3} \rightarrow y_{3}$. Different functions (14) and (15) are used as $y_{1}$ and $y_{2}$ generally have much smaller amplitudes than $y_{3}$.

\section{B. Identification of Transfer Function Matrix}

To estimate the transfer function matrix, one steady-state period of the outputs $y_{1}, y_{2}$, and $y_{3}$ which are corrupted by noise and nonlinear distortion (see Fig. 4) is used. Contributions from $x_{1}, x_{2}$, and $x_{3}$ are first separated at each of the outputs by extracting the output power only at harmonics present at any particular input. For example, to find the contribution of $x_{1}$ to $y_{1}$, the harmonics $1,5,7,11,13,17, \ldots$, corresponding to Signal B are extracted, to find the contribution of $x_{2}$ to $y_{1}$, the harmonics $2,10,14,22,26,34, \ldots$, corresponding to Signal $E$ are extracted, and to find the contribution of $x_{3}$ to $y_{1}$, the harmonics $3,9,15$, $21,27,33, \ldots$, corresponding to Signal $\mathrm{F}$ are extracted. This is shown in Fig. 5, where the decoupling of the effects of different inputs can be observed. However, it should be noted that the channel noise cannot be decoupled, because the noise signals contain all harmonics except harmonic 0 .

In the subsequent step, the frequency domain data are fitted using the Estimator for Linear Systems (ELiS) [26] in the Frequency Domain System Identification Toolbox. To avoid the effects of aliasing, the ELiS estimation procedure is applied only to harmonics $\leq 67$, corresponding to frequencies less than 0.4 times the sampling frequency. 

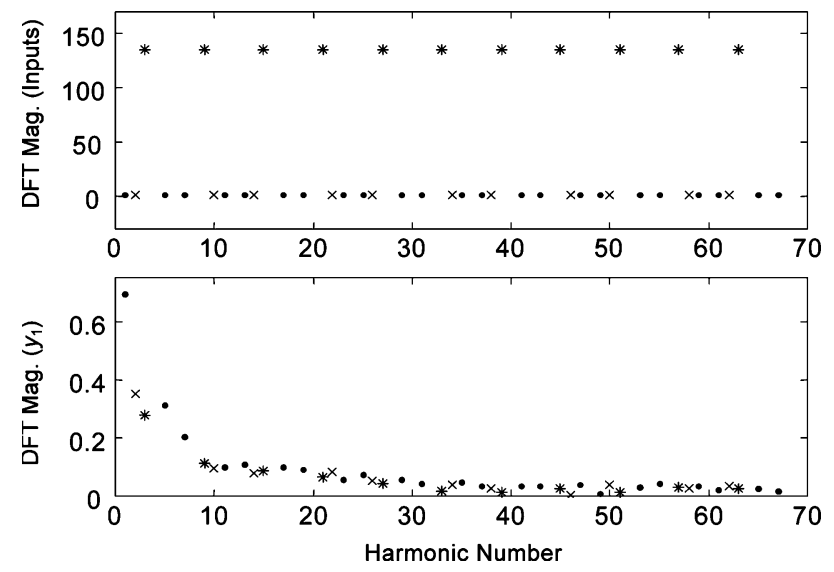

Fig. 5. DFT magnitudes of the inputs (top) and the signal $y_{1}$ (bottom) with the contributions of $x_{1}$ (dots), $x_{2}$ (crosses) and $x_{3}$ (asterisks) separated. Only harmonics $\leq 67$, corresponding to frequencies less than 0.4 times the sampling frequency, are shown.

With this procedure, the transfer function matrix of the distillation column is estimated to be

$$
\begin{aligned}
& \hat{G}_{\text {PRML }}(\mathrm{s}) \\
& =\left[\begin{array}{ccc}
\frac{0.67 \mathrm{e}^{-2.6 \mathrm{~s}}}{6.71 \mathrm{~s}+1} & \frac{-0.54 \mathrm{e}^{-3.6 \mathrm{~s}}}{7.24 \mathrm{~s}+1} & \frac{-0.0069 \mathrm{e}^{-0.9 \mathrm{~s}}}{13.63 \mathrm{~s}+1} \\
\frac{1.23 \mathrm{e}^{-6.5 \mathrm{~s}}}{3.29 \mathrm{~s}+1} & \frac{-2.42 \mathrm{e}^{-3.0 \mathrm{~s}}}{4.60 \mathrm{~s}+1} & \frac{-0.0085 \mathrm{e}^{-1.1 \mathrm{~s}}}{6.75 \mathrm{~s}+1} \\
\frac{-34.08 \mathrm{e}^{-9.1 \mathrm{~s}}}{8.56 \mathrm{~s}+1} & \frac{48.60 \mathrm{e}^{-9.3 \mathrm{~s}}}{10.65 \mathrm{~s}+1} & \frac{0.91(12.30 \mathrm{~s}+1) \mathrm{e}^{-1.1 \mathrm{~s}}}{(3.91 \mathrm{~s}+1)(18.48 \mathrm{~s}+1)}
\end{array}\right] .
\end{aligned}
$$

\section{Comparison With Two Other Signal Sets}

A well established method to apply pseudo-random signals for MIMO identification is to start with an original signal and then modulate the signal using rows of the Hadamard matrix to generate uncorrelated signals based on the original signal [10]. Four periods of a near-binary pseudo-random signal called a quadratic residue ternary (QRT) signal of original period 41 are cascaded to form the input $x_{1}$, such that $N=164$. The inputs $x_{2}$ and $x_{3}$ are generated by multiplying the elements of the signal to $x_{1}$ by $[+1-1+1-1 \cdots]$ and $[+1+1-1-1 \cdots]$, respectively. All the signals are scaled to the amplitudes as given in Section VII-A. The sampling interval is maintained at $0.5 \mathrm{~min}$. Using this technique, the transfer function matrix is identified as

$$
\begin{aligned}
& \hat{G}_{\mathrm{QRT}}(\mathrm{s}) \\
& =\left[\begin{array}{ccc}
\frac{0.65 \mathrm{e}^{-2.6 \mathrm{~s}}}{6.47 \mathrm{~s}+1} & \frac{-0.63 \mathrm{e}^{-3.5 \mathrm{~s}}}{8.77 \mathrm{~s}+1} & \frac{-0.0049 \mathrm{e}^{-1.0 \mathrm{~s}}}{9.67 \mathrm{~s}+1} \\
\frac{1.55 \mathrm{e}^{-6.5 \mathrm{~s}}}{4.68 \mathrm{~s}+1} & \frac{-2.47 \mathrm{e}^{-3.0 \mathrm{~s}}}{4.81 \mathrm{~s}+1} & \frac{-0.0093 \mathrm{e}^{-1.3 \mathrm{~s}}}{6.78 \mathrm{~s}+1} \\
\frac{-30.52 \mathrm{e}^{-9.2 \mathrm{~s}}}{6.93 \mathrm{~s}+1} & \frac{53.02 \mathrm{e}^{-9.4 \mathrm{~s}}}{11.79 \mathrm{~s}+1} & \frac{1.09(14.59 \mathrm{~s}+1) \mathrm{e}^{-1.0 \mathrm{~s}}}{(3.61 \mathrm{~s}+1)(29.69 \mathrm{~s}+1)}
\end{array}\right] .
\end{aligned}
$$

Another well-established method, described in [9] as the input phase separation method, is to use appropriately delayed versions of a PRBS as the system inputs. This method requires a considerably longer measurement period than the two other methods described above, because it is necessary for the output responses due to different input phases to be separable in the input-output crosscorrelation function. Shifted versions of a PRBS of period $N=511$ are used to perturb the system,
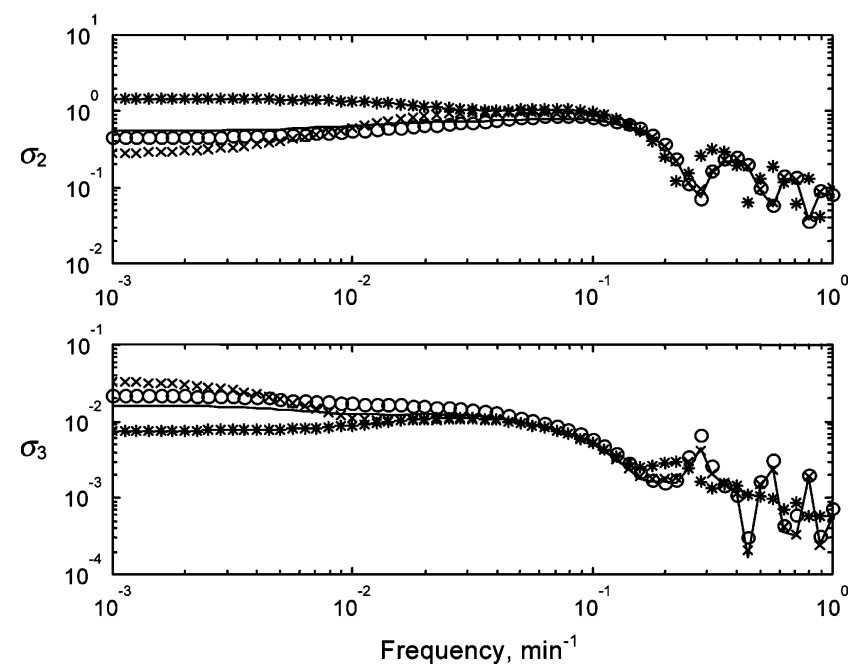

Fig. 6. Singular values $\sigma_{2}$ and $\sigma_{3}$ of the gain matrix. Solid line: Theoretical; Circles: Proposed PRML; Crosses: Hadamard-modulated QRT; Asterisks: Shifted PRBS.

with the optimal shifts calculated based on the knowledge of the approximate individual time delays of each of the transfer functions in $G(s)$. This method gives

$$
\begin{aligned}
& \hat{G}_{\operatorname{PRBS}}(\mathrm{s}) \\
& =\left[\begin{array}{ccc}
\frac{0.67 \mathrm{e}^{-2.6 \mathrm{~s}}}{7.06 \mathrm{~s}+1} & \frac{-0.61 \mathrm{e}^{-3.5 \mathrm{~s}}}{7.95 \mathrm{~s}+1} & \frac{-0.0049 \mathrm{e}^{-1.0 \mathrm{~s}}}{8.69 \mathrm{~s}+1} \\
\frac{1.27 \mathrm{e}^{-6.5 \mathrm{~s}}}{3.38 \mathrm{~s}+1} & \frac{-3.04 \mathrm{e}^{-3.0 \mathrm{~s}}}{5.46 \mathrm{~s}+1} & \frac{-0.0081 \mathrm{e}^{-1.1 \mathrm{~s}}}{6.79 \mathrm{~s}+1} \\
\frac{-42.91 \mathrm{e}^{-9.1 \mathrm{~s}}}{10.15 \mathrm{~s}+1} & \frac{37.50 \mathrm{e}^{-10.0 \mathrm{~s}}}{9.77 \mathrm{~s}+1} & \frac{0.81(11.22 \mathrm{~s}+1) \mathrm{e}^{-1.0 \mathrm{~s}}}{(4.10 \mathrm{~s}+1)(15.13 \mathrm{~s}+1)}
\end{array}\right] .
\end{aligned}
$$

\section{Identification of Singular Values of Gain Matrix}

According to Rasmussen and Jørgensen [27], and Li and Lee [28], for model-based control, it is very important to identify accurately the singular values of the gain matrix. The estimated values of $\sigma_{2}$ and $\sigma_{3}$ are shown in Fig. 6, where it can be observed that the proposed method gives better estimates overall. For $\sigma_{1}$, all three methods give accurate estimates and these are therefore not shown.

\section{CONCLUSION}

An approach to designing ternary pseudo-random signals from Galois fields, suitable for application in the identification of MIMO systems, has been described. The signal sets possess the "zippered spectrum" and are designed using the harmonic properties of their primitive signals. The approach takes advantage of the larger number of possible combinations when allowing multiple periods within a period of the primitive signal. The signals can be designed to have uniform nonzero harmonics. Signals with good immunity to the effects of noise and nonlinear distortion can be easily selected from the design Table provided. An application has been presented for an ill-conditioned three-input three-output distillation column. For this application, the proposed signal set resulted in better estimates of the singular values of the gain matrix, compared with Hadamard-modulated QRT signals and shifted PRBS signals, despite the total experimentation time using the proposed method being only $1 / 3$ of that of the shifted PRBS technique. 


\section{REFERENCES}

[1] J. MacWilliams, "An example of two cyclically orthogonal sequences with maximum period," IEEE Trans. Inf. Theory, vol. 13, no. 4, pp. 338-339, Apr. 1967.

[2] P. A. N. Briggs and K. R. Godfrey, "Design of uncorrelated signals," Electron. Lett., vol. 12, no. 21, pp. 555-556, 1976

[3] M. El-Fandi, I. A. Henderson, J. McGhee, and P. McGlone, "Uncorrelated multisymbol signals for MIMO system identification," IEEE Trans. Instrum. Meas., vol. 47, no. 10, pp. 1133-1138, Oct. 1998.

[4] D. E. Rivera, S. Zong, and W. M. Ling, "A control-relevant multivariable system identification methodology based on orthogonal multifrequency input perturbations," in Proc. 11th IFAC Symp. Syst. Identification, Fukuoka, Japan, Jul. 1997, pp. 595-600.

[5] D. E. Rivera, H. Lee, H. D. Mittelmann, and M. W. Braun, "High-purity distillation: Using plant-friendly multisine signals to identify a strongly interactive process," IEEE Control Syst. Mag., vol. 27, no. 5, pp. 72-89, Oct. 2007.

[6] H. Lee, "A plant-friendly multivariable system identification framework based on identification test monitoring," Ph.D. dissertation, Dept. Chem. Eng., Arizona State Univ., Tempe, AZ, 2006.

[7] H. A. Barker and K. R. Godfrey, "System identification with multilevel periodic perturbation signals," Control Eng. Practice, vol. 7, no. 6, pp. 717-726, 1999.

[8] A. H. Tan and K. R. Godfrey, "Modeling of direction-dependent processes using Wiener models and neural networks with nonlinear output error structure," IEEE Trans. Instrum. Meas., vol. 53, no. 3, pp. 744-753, Jun. 2004

[9] P. A. N. Briggs and K. R. Godfrey, "Pseudorandom signals for the dynamic analysis of multivariable systems," Proc. IEE, vol. 113, no. 7, pp. 1259-1267, 1966.

[10] J. Utsal, "Pseudo-random binary noise for system identification," $\mathrm{Ph} . \mathrm{D}$. dissertation, Dept. Eng., Univ. Cambridge, Cambridge, U.K., 1965.

[11] H. A. Barker, A. H. Tan, and K. R. Godfrey, "Ternary input signal design for system identification," IET Control Theory Appl., vol. 1, no. 5, pp. 1224-1233, 2007.

[12] C. Evans, D. Rees, and L. Jones, "Nonlinear disturbance errors in system identification using multisine test signals," IEEE Trans. Instrum. Meas., vol. 43, no. 4, pp. 238-244, Apr. 1994.

[13] C. Evans, D. Rees, and L. Jones, "Identifying linear models of systems suffering nonlinear distortions, with a gas turbine application," IEE Proc.-Control Theory Appl., vol. 142, no. 3, pp. 229-240, 1995.

[14] J. Schoukens, T. Dobrowiecki, and R. Pintelon, "Parametric and nonparametric identification of linear systems in the presence of nonlinear distortions-A frequency domain approach," IEEE Trans. Autom. Control, vol. 43, no. 2, pp. 176-190, Feb. 1998.
[15] D. E. Rivera, H. Lee, H. D. Mittelmann, and M. W. Braun, "Constrained multisine input signals for plant-friendly identification of chemical process systems," J. Process Control, vol. 19, no. 4, pp. 623-635, 2009.

[16] H. A. Barker, "Design of multi-level pseudo-random signals for system identification," in Perturbation Signals for System Identification, K. Godfrey, Ed. Hemel Hempstead, U.K.: Prentice-Hall, 1993.

[17] H. A. Barker, "Primitive maximum-length sequences and pseudorandom signals," Trans. InstMC, vol. 26, no. 4, pp. 339-348, 2004.

[18] A. H. Tan, K. R. Godfrey, and H. A. Barker, "Design of computer-optimised pseudo-random maximum length signals for linear identification in the presence of nonlinear distortions," IEEE Trans. Instrum. Meas., vol. 54, no. 12, pp. 2513-2519, Dec. 2005.

[19] K. R. Godfrey, H. A. Barker, and A. J. Tucker, "Comparison of perturbation signals for linear system identification in the frequency domain," IEE Proc.-Control Theory Appl., vol. 146, no. 6, pp. 535-548, 1999.

[20] M. F. L. Foo, A. H. Tan, and K. P. Basu, "Maximum length ternary signal design based on Nyquist point mapping," in Proc. 14th IFAC Symp. Syst. Identification, Newcastle, Australia, Mar. 2006, pp. $1127-1132$

[21] A. H. Tan, "Design of truncated maximum length ternary signals where their squared versions have uniform even harmonics," IEEE Trans. Autom. Control, vol. 52, no. 5, pp. 957-961, May 2007.

[22] A. H. Tan and M. F. L. Foo, "Ternary pseudorandom signal design for uniform excitation and reduced effect of nonlinear distortion," Electron. Lett., vol. 42, no. 12, pp. 676-678, 2006.

[23] Y.Zhu and P. Stec, "Simple control-relevant identification test methods for a class of ill-conditioned processes," J. Process Control, vol. 16, no. 10, pp. 1113-1120, 2006.

[24] A. Micchi and G. Pannocchia, "Comparison of input signals in subspace identification of multivariable ill-conditioned systems," $J$. Process Control, vol. 18, no. 6, pp. 582-593, 2008.

[25] B. A. Ogunnaike, J. P. Lemaire, M. Morari, and W. H. Ray, "Advanced multivariable control of a pilot-plant distillation column," Amer. Inst. Chem. Eng. J., vol. 29, no. 4, pp. 632-640, 1983.

[26] I. Kollár, Frequency Domain System Identification Toolbox for Use with MATLAB. Natick, MA: The MathWorks Inc., 1994.

[27] K. H. Rasmussen and S. B. Jørgensen, "Parametric uncertainty modelling for robust control: A link to identification," Comput. Chem. Eng., vol. 23 , no. 8, pp. $987-1003,1999$.

[28] W. Li and J. H. Lee, "Control relevant identification of ill-conditioned systems: Estimation of gain directionality," Comput. Chem. Eng., vol. 20, no. 8, pp. 1023-1042, 1996. 